\title{
PREDICTION OF HEMORRHAGIC TRANSFORMATION WITH BLOOD BIOMARKERS IN THE ERA OF MECHANICAL THROMBECTOMY
}

Alejandro Bustamante, Anna Penalba, Teresa García-Berrocoso, Caty Carrera, Oriol Ventura, Marc Fernández, Marta Rubiera, Eva Baldrich, Mónica Millán, Antoni Serrano-Blanco, Jaume Roquer, Antoni Dávalos, Israel Fernández-Cadenas, Joan Montaner

The PRE-test-STROKE [Personalizing REperfusion Therapeutic Strategies in Stroke] investigators

\section{INTRODUCTION}

- After more than two decades of experience with intravenous tissue-type plasminogen activator (IV-tPA), hemorrhagic transformation (HT) has reduced dramatically, with current rates lower than 4\% (ECASS definition) or 2\% (SITS-MOST definition) [1]

- Different pathophysiological mechanisms might be related with HT after mechanical thrombectomy (MT), what is suggested by different patterns, such as subarachnoid hemorrhages, not always related with direct catheter injury [2], or antiplatelet-related HT in patients receiving stenting for tandem occlusions [3].

- We aimed to test whether blood biomarkers previously associated with HT after IV-tPA are still useful for that purpose, in both patients treated with IV-tPA and IV-tPA + MT

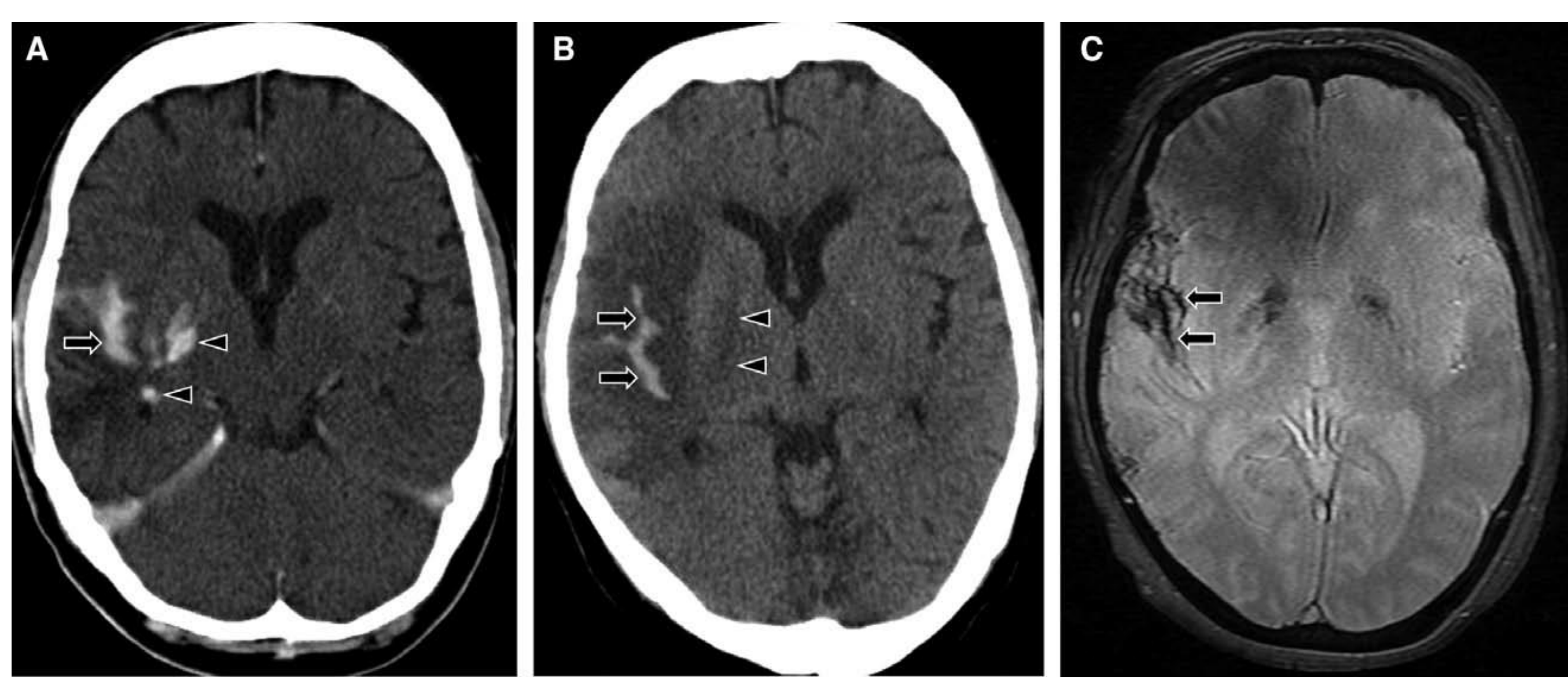

Subarachnoid hemorrhage after MT, from Yoon et al, Stroke 2013

\section{METHODS}

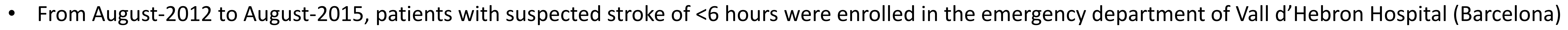

- Blood samples were collected before treatment

- Patients treated with IV-tPA were selected

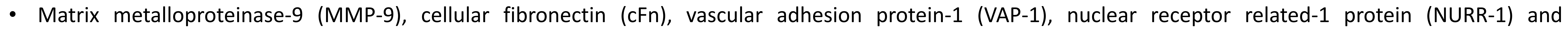
carcinoembrionary antigen cell adhesion molecule-1 (CEACAM-1) were measured by ELISA

- Primary outcome was parenchymal hematoma (PH)

- Secondary outcomes were any HT and symptomatic intracerebral hemorrhage (sICH) according ECASS criteria

- The analysis was performed in all patients treated just IV-tPA first, and after exclusion of patients treated with IV-tPA + MT

\section{RESULTS}

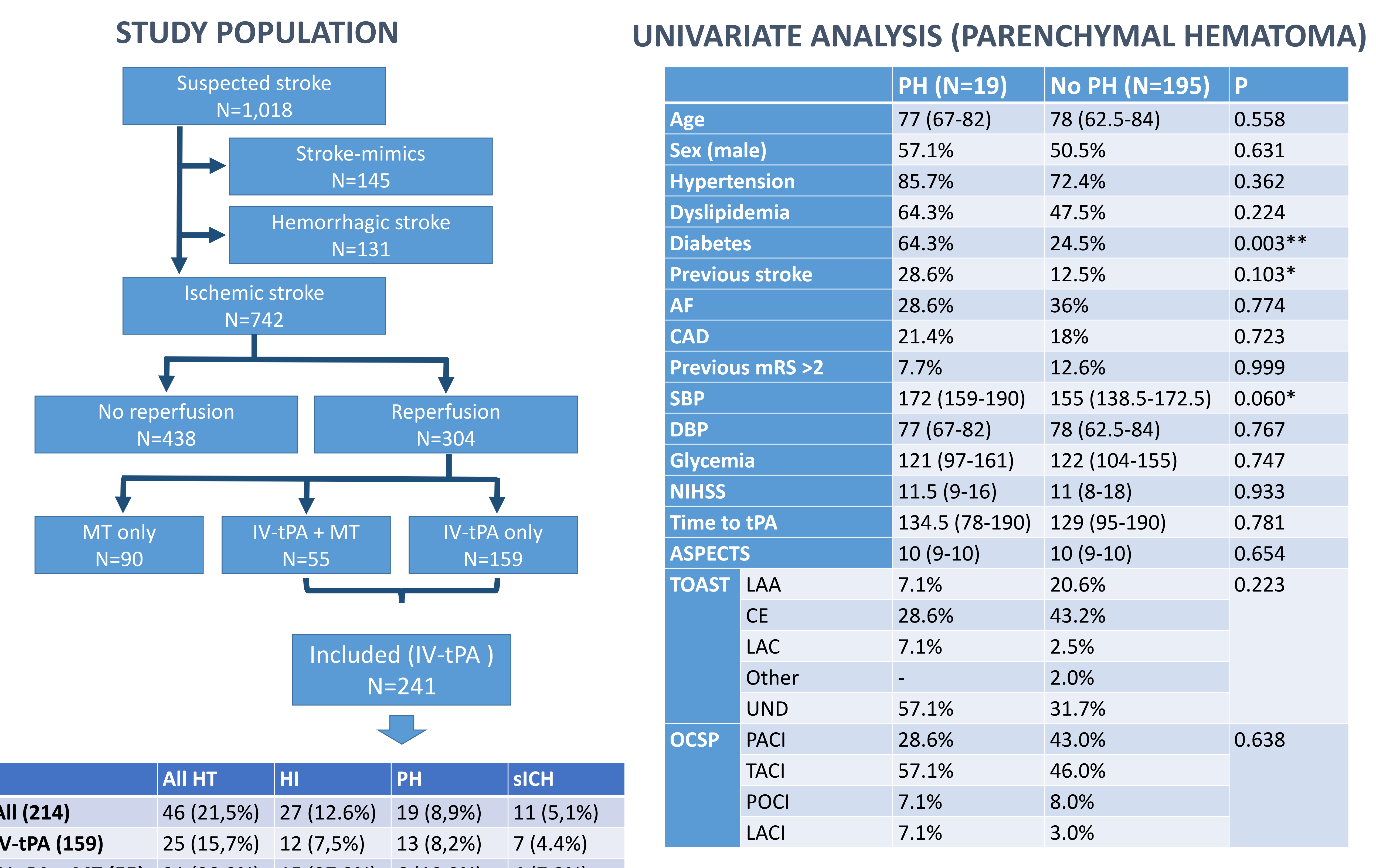

BIOMARKERS AND PARENCHYMAL HEMATOMA

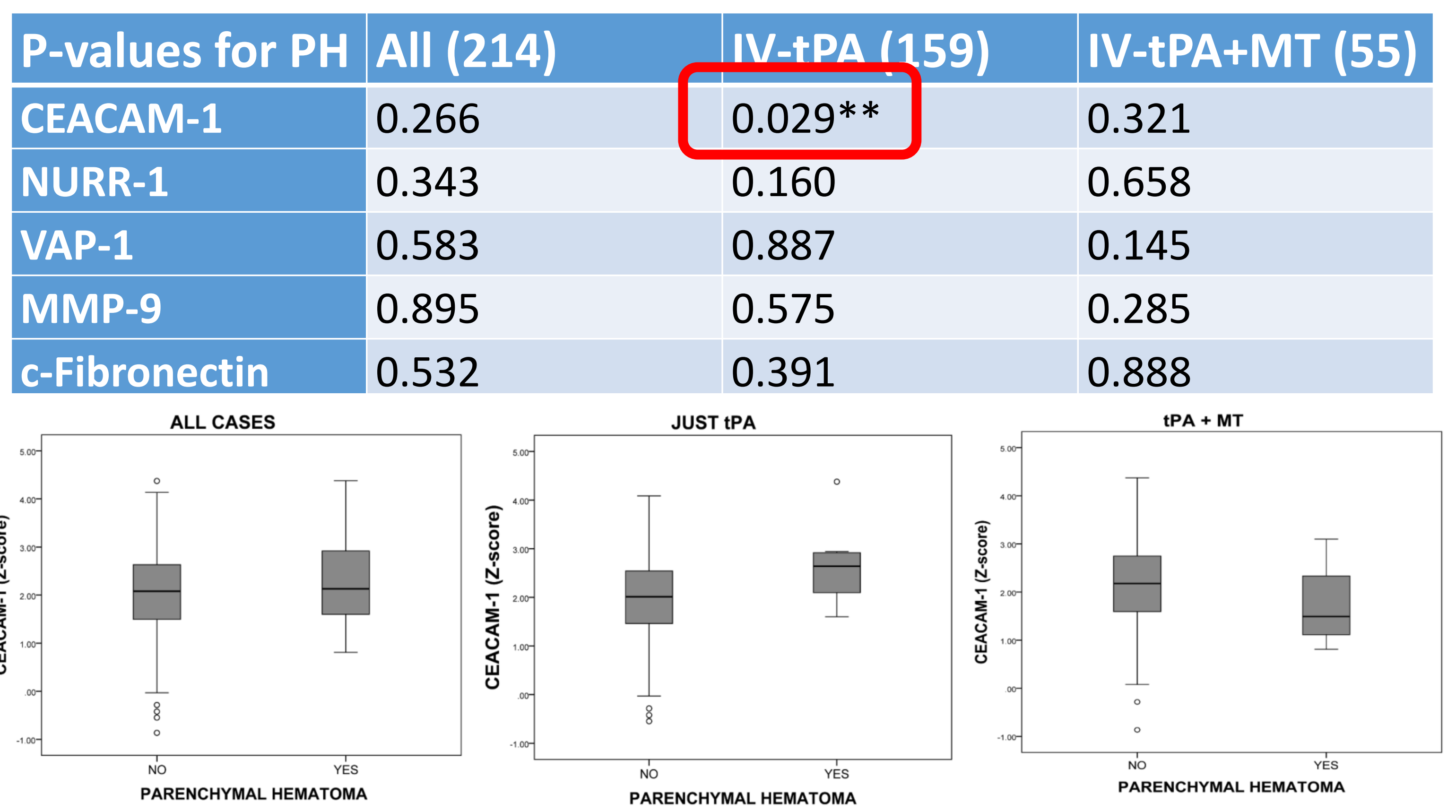

LOGISTIC REGRESSION FOR PH, JUST IV-tPA

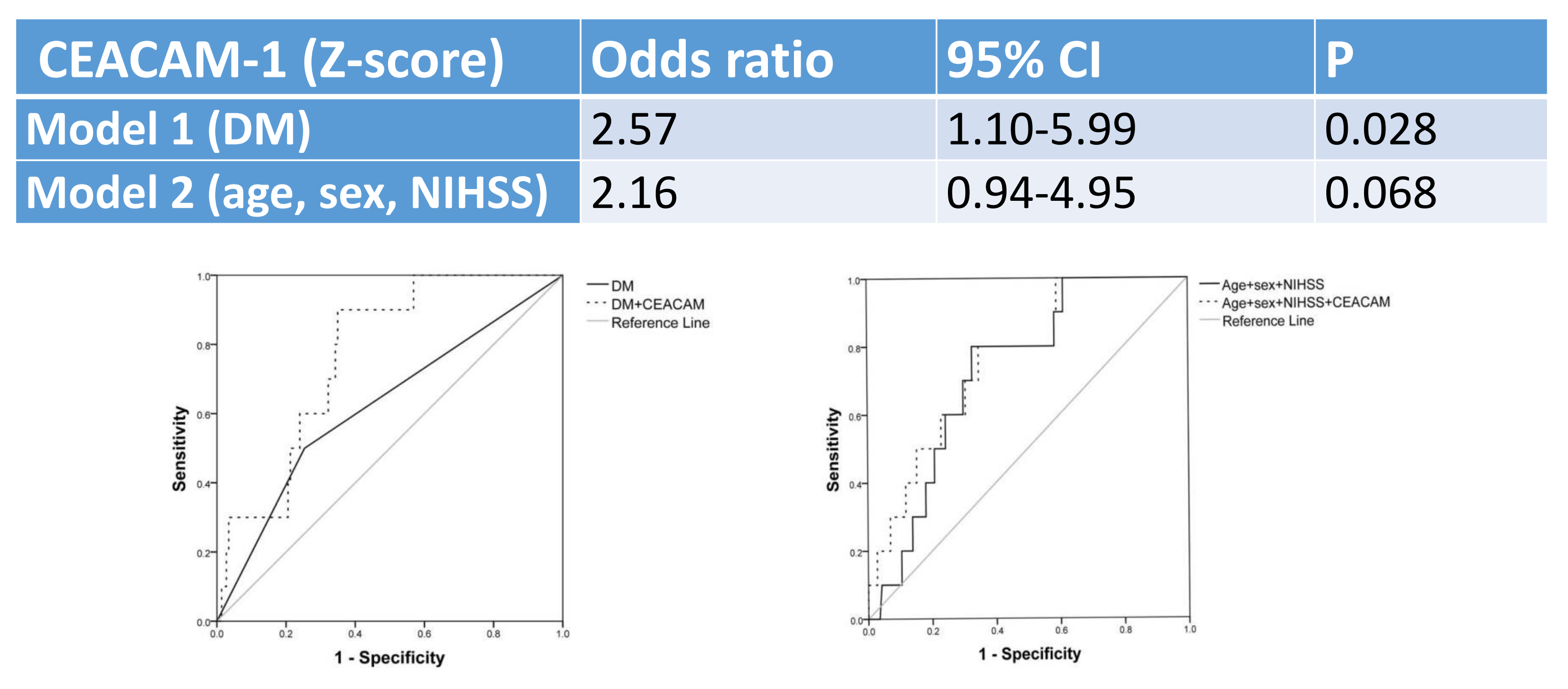

\section{CONCLUSIONS}

- CEACAM-1 might be a predictor of tPA-related PH. However, it was not further significant when including patients treated with IV-tPA + MT

- Biomarkers previously associated with HT were not replicated in this prospective cohort. Very low rates of HT and different mechanisms in endovascular procedures might be responsible for that

- HT prediction in IV-tPA treated patients might be of little help in the era of MT. Further research effort might be focused towards other indications, such as recanalization after IV-tPA or futile recanalization after MT

\section{REFERENCES}

1. Capellari M, et al. Stroke 2018; 49: 397-404

2. Yoon W, et al. Stroke 2013;44:414-419

3. Heck DV, et al. J Neurointerv Surg. 2015;7:170-5
CONTACT

alejandro.bustamante@vhir.ors

www.lin-ben.com

www.stroke-biomarkers.com

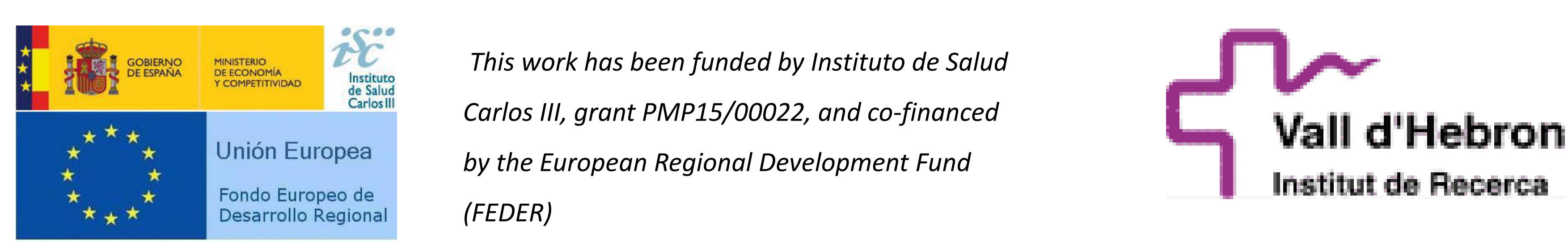

